\title{
Isolated Sphenoid Sinus Mucocele: A Rare Case and Review of Literature
}

\author{
${ }^{1}$ Karan Gupta, ${ }^{2}$ Ramandeep Singh Virk, ${ }^{3}$ Satheesh Kumar Sunku
}

\section{ABSTRACT}

Isolated sphenoid sinus mucoceles are uncommon and difficult to diagnose clinically owing to the inaccessibility of the sphenoid sinus to clinical examination. A case of infected sphenoid sinus mucocele in which the patient complained of progressive nasal obstruction and postnasal drip without any other classical features of sphenoid sinus mucocele is discussed here. The pathology of mucocele and endoscopic sinus surgery as the treatment has been discussed in this article.

Keywords: Sphenoid sinus, Mucocele, Endoscopic marsupialization.

How to cite this article: Gupta K, Virk RS, Sunku SK. Isolated Sphenoid Sinus Mucocele: A Rare Case and Review of Literature. J Postgrad Med Edu Res 2015;49(2):91-93.

\section{Source of support: Nil}

Conflict of interest: None

\section{INTRODUCTION}

Mucoceles are benign, encapsulated, expansile, locally invasive masses within a paranasal sinus filled with mucus and lined by epithelium. They occur in all of the paranasal sinuses but arise most commonly in the frontal sinus. Isolated sphenoid sinus mucocele is rare and often misdiagnosed. ${ }^{1}$ Since its first description by Berg in 1889 less than 150 cases have been reported in the medical literature. ${ }^{2}$

The presenting symptoms and signs of sphenoid sinus mucocele are related to its anatomical position as well as its adjacent structures. ${ }^{2}$ Headache being the most common, ophthalmologic symptoms, cranial nerve deficits, nasal blockage and rhinorrhea are the other frequent presentations. Treatment of sphenoid sinus mucocele is surgery. Though traditionally various routes like septal, antral, ethmoidal, intranasal or intracranial have been described, introduction of endoscopic sinus surgery is presently widely advocated as the treatment modality.

\footnotetext{
${ }^{1,3}$ Senior Resident, ${ }^{2}$ Assistant Professor

${ }^{1-3}$ Department of ENT, Postgraduate Institute of Medical Education and Research, Chandigarh, India
}

Corresponding Author: Satheesh Kumar Sunku, Senior Resident, Department of ENT, Postgraduate Institute of Medical Education and Research, Chandigarh, India, e-mail: pgient@rediffmail.com

\section{CASE REPORT}

A 55-year-old male presented to the outdoor department of Otolaryngology Head and Neck Surgery at the Postgraduate Institute of Medical Education and Research, Chandigarh, India, with the chief complaint of progressive nasal obstruction right side for 1 year. Nasal obstruction was more during night and associated with postnasal drip occasionally. There were no features suggestive of allergic rhinosinusitis like recurrent sneezing, nasal discharge, facial pain or headache. No other comorbidity or addictions were noted. Throat examination showed postnasal discharge on posterior pharyngeal wall. Nasal endoscopy revealed smooth mucosal bulge in nasopharynx more on right side. Noncontrast computed tomography (CT) showed homogenous, expansile softtissue density lesion involving total sphenoid sinus projecting into nasopharynx more on right side (Figs 1A and B).

Patient was taken up for endoscopic surgery and, intraoperatively, there was a smooth mucosa covered bulge in nasopharynx. On incising the mucosa thick, greenish fluid approximately $15 \mathrm{ml}$ was drained, which was filling the sphenoid cavity and expanding all walls of the sinus. There were greenish granulations carpeting posterior wall, which were removed. Bilateral optic nerve and internal carotid artery were found dehiscent. Gram staining was sterile, fungal smear from the secretions showed no growth of hyphae. Postoperative histopathological examination report showed sphenoidal mucocele. Postoperative follow-up at 3 months showed well-healed healthy cavity (Figs 2A and B).

\section{DISCUSSION}

Mucoceles occur most commonly in the frontal and ethmoid sinuses but are quite infrequent in the sphenoid sinus representing less than $2.0 \%$ of all paranasal sinus mucoceles. ${ }^{3}$ Sphenoid sinus is a rudimentary cavity at birth and enlarges to its adult size in early teens. Several important structures located adjacent to the sinus may be compressed by an expanding mucocele which gives rise to a variety of clinical symptoms. Sphenoid mucocele occurs as early as in 2nd decade and up to the 7th decade of life.

The pathogenesis of mucocele in paranasal sinuses is uncertain and is believed to be multifactorial. Three mechanisms have been put forward: ostium obstruction, 

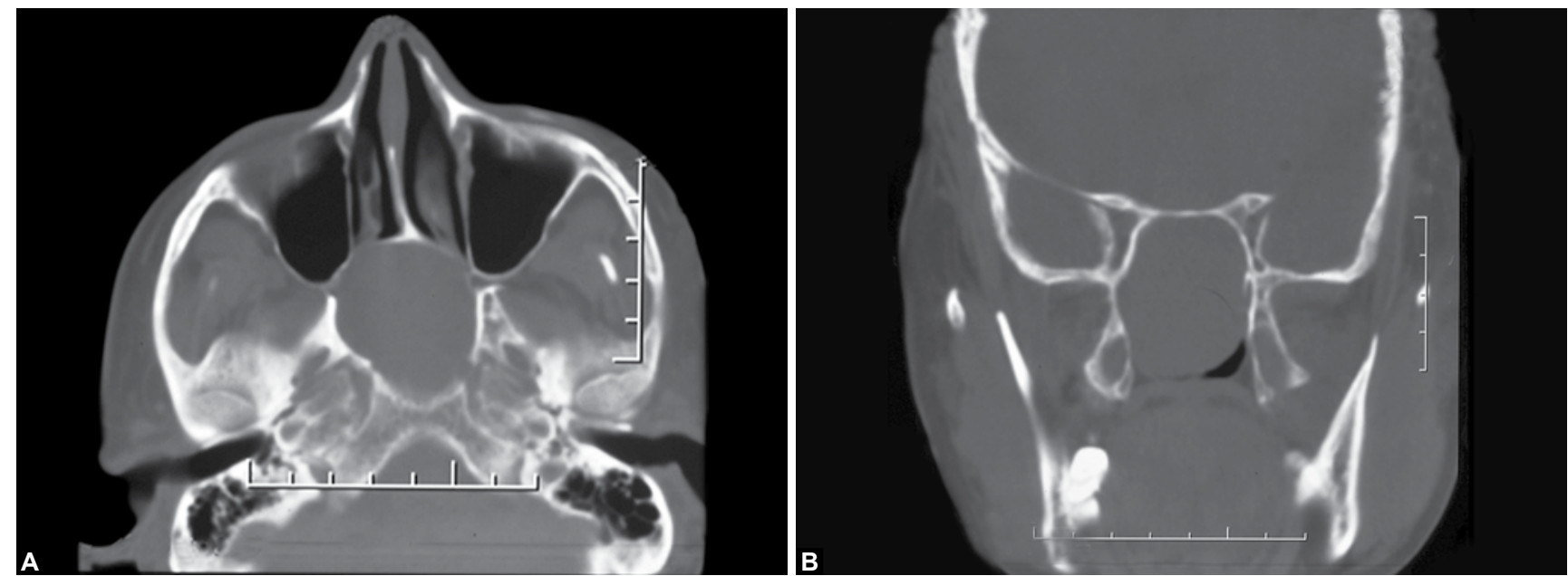

Figs 1A and B: (A) Noncontrast computed tomography axial cut and (B) coronal cut showing a homogenous soft-tissue density completely involving the sphenoid sinus and causing expansion

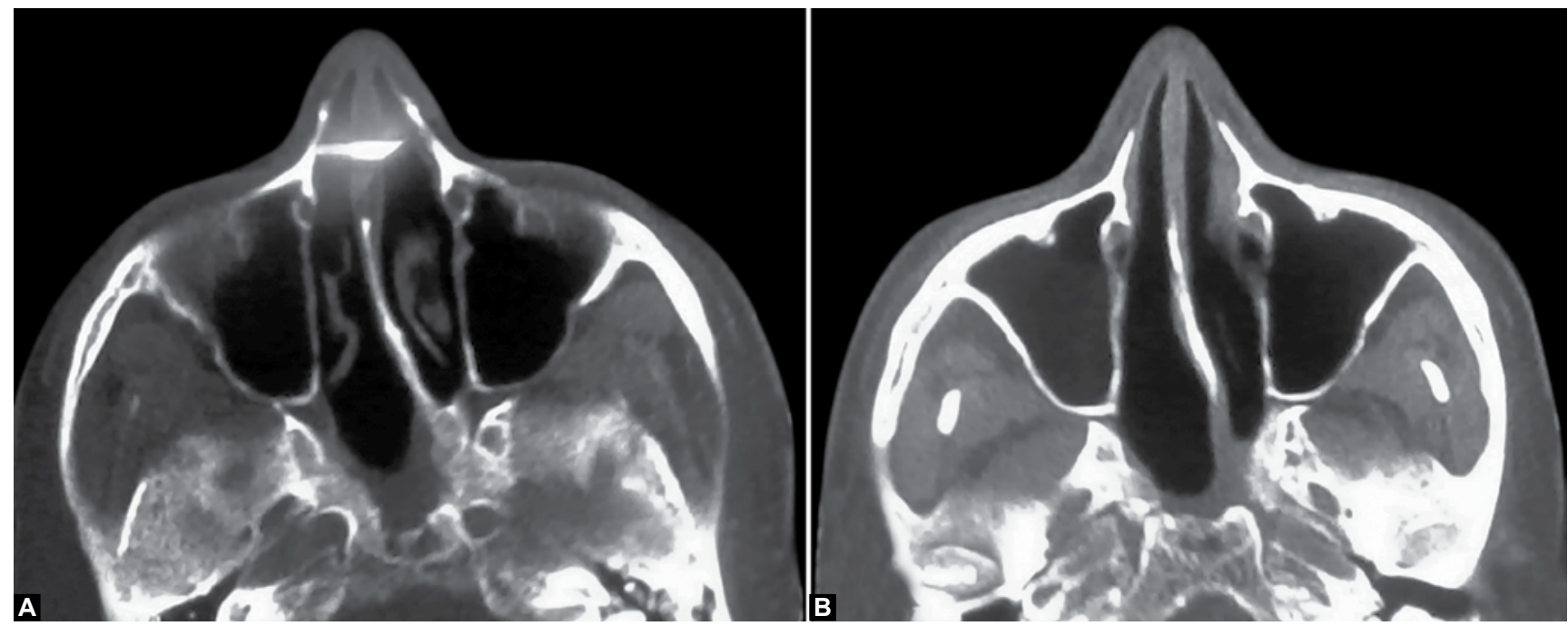

Figs 2A and B: NCCT axial cut done 3 months postoperative showing well-healed sphenoidal cavity

secretory gland obstruction and submucosal edema. The most common mechanism is ostium obstruction due to chronic infection, polyposis, tumor and previous surgery. Obstruction to the duct of secretory gland will result in a retention cyst which may expand into a mucocele. Submucosal edema due to allergic inflammation can cause ostium blockage with mucocele formation. ${ }^{2,4,5}$ As it expands, the sphenoid mucocele spreads along least resistance into posterior ethmoid sinus forming sphenoethmoidal mucocele.

Diagnosis of sphenoid mucocele may pose a problem because of its rarity and relative inaccessibility to clinical examination. Retro-orbital headache is the most common presenting symptom followed by ocular disturbances comprising internal and external ophthalmoplegia, visual deficit and unilateral exophthalmos can occur. Compression of trigeminal nerve divisions may cause secondary neuralgia and present as facial pain. Otolaryngological symptoms of nasal obstruction and rhinorrhea are seen in about $50 \%$ of the patients. ${ }^{4,6}$
The neoplasms arising from pituitary region must be consider as differential diagnosis, such as craniopharyngioma, meningioma, glioma, intracranial chordoma, pituitary gland tumor and nasopharyngeal carcinoma. Radiological investigations can differentiate these conditions from sphenoid sinus mucocele. A sphenoid sinus mucocele appears as an opacified, expanded sinus on CT. ${ }^{1}$ Magnetic resonance imaging (MRI) may be helpful for differentiating mucocele from other lesions that expand the sinus and have similar density on CT. Magnetic resonance imaging typically demonstrates a homogeneous expansile hypointense lesion on T1-weighted images with hyperintense T2 signal. Because of variable protein content within long-standing mucoceles, signal intensity can be highly variable on both T1- and T2-weighted sequences. ${ }^{7}$ Radiologically, a chromophobe adenoma is most likely to simulate sphenoid sinus mucocele. ${ }^{4}$

The treatment of sphenoid mucocele is to fenestrate the sinus wall and marsupialize the sinus mucosa. ${ }^{8}$ 
Traditionally, there are several surgical approaches to the sphenoid sinus. Trans-septal approaches via either sublabial or intranasal incision are commonly used. These approaches injure the septum and the surgical access may be inadequate to provide the wide-open drainage necessary. Transnasal route with the use of operating microscope is a direct approach to the sphenoid sinus but it frequently necessitates dissection of the ethmoid sinus and turbinates to obtain a good exposure. External or transnasal sphenoethmoidectomy requires removal of the ethmoid sinus air cells to gain access to the sphenoid sinus with increased morbidity and risks of damage to the orbit, optic nerve and skull-base. Intraoral transpalatal approach requires extensive dissection.

Endoscopic sinus surgery has been found to be the most efficacious in the treatment of sphenoid mucocele ${ }^{2,8}$ in which the sphenoid can be approached directly. Endoscope provides excellent visualization of the operative field with adequate magnification. This approach obviates the extensive dissection required in most of the traditional approaches and does not require external incision. Minimal blood loss and reduced operative time are the advantages. Furthermore, endoscopic sinus surgery can also be done under local anesthesia in those where general anesthesia is contraindicated.

However, endoscopic sinus surgery is not devoid of complications including both anesthetic and surgical with an estimated rate between 2 and $17 \%$. These include cerebrospinal fluid leak and intracranial injury from inadvertent entry into skull base, blindness due to damage to the optic nerve, diplopia from injury to occulomotor and trochlear nerves and nasolacrimal duct injury. ${ }^{8}$ Fatal hemorrhage can occur due to injury to internal carotid artery or an undiagnosed aneurysmal $\mathrm{ICA}^{9}$ or on displacing the attachment of intersinus septum over already dehiscent artery. A good radiological work up before surgery will definitely help to avoid this complication. Overall complication rate can be reduced by proper training in the endoscopic surgeries, increased vigilance and careful surgical dissection with compulsive postoperative care. ${ }^{10}$

\section{CONCLUSION}

Isolated sphenoid sinus mucocele is a rare entity and only less than $50 \%$ cases are associated with the classical symptoms. A strong suspicion and a routine nasal endoscopy will provide great information to diagnose the condition. Endoscopic sinus surgery to marsupialize the mucocele is the best modality of treatment in view of the direct surgical access, good visualization and low morbidity.

\section{REFERENCES}

1. Chui Me, Briant TDR, Gray T, Horsey WJ, Hudson AR, Tucker W. Computed tomography of sphenoid sinus mucocele. J Otolaryngol 1983;12(4):263-269.

2. Stankiewicz JA. Sphenoid sinus mucocele. Arch Otolaryngol Head Neck Surg 1989;115(6):735-740.

3. Darouassi Y, Righini CA, Reyt E. Mucoceles of the sphenoid sinus: a report of four cases and review of the literature. BENT 2005;1(4):181-185.

4. Weaver RG, Gates GA. Mucoceles of the sphenoid sinus. Otolaryngol Head Neck Surg 1979;87(2):168-173.

5. Tooriuni DM, Berktold RE. Multiple frontoethmoid mucoceles. Ann Otol Rhinol Laryngol 1989;98(10):831-832.

6. Close LG. OCorner WE. Sphenoethmoidal mucoceles with intracranial extension. Otolaryngol Head Neck Surg 1983;91(4):350-357.

7. Dawson RC III, Horton JA. MR imaging of mucoceles of the sphenoid sinus. AJNR 1989;10(3):613-614.

8. Kennedy DW, Josephson JS, Zinreich SJ, Mattox DE, Goldsmith MM. Endoscopic sinus surgery for mucoceles: a viable alternative. Laryngoscope 1989;99(9):885-895.

9. Maniglia AJ. Fatal and major complications secondary to nasal and sinus surgery. Laryngoscope 1989;99(3):276-283.

10. Stankwiecz JA. Complications of endoscopic sinus surgery. Otolaryngol Clin North Am 1989;22(4):749-758. 